\title{
ATTRIBUte REDUCTION AND DECISION TREE PRUNING To SiMPLIFY LIVER FibROSIS Prediction Algorithms A COHORT STUdY
}

\author{
Mahasen Mabrouk ${ }^{1}$, Abubakr Awad ${ }^{2}$, Hend Shousha ${ }^{1}$, Wafaa Alakel ${ }^{1,3}$, \\ Ahmed Salama ${ }^{1}$, Tahany Awad ${ }^{1}$ \\ ${ }^{1}$ Endemic Medicine and Hepatology Department, Faculty of Medicine, Cairo \\ University, Cairo, Egypt \\ ${ }^{2}$ School of Natural and Computing Sciences, University of Aberdeen, Aberdeen, \\ UK \\ ${ }^{3}$ National Hepatology and Tropical Medicine Research Institute, Ministry of \\ Health and Population, Cairo, Egypt
}

\begin{abstract}
Background: Assessment of liver fibrosis is a vital need for enabling therapeutic decisions and prognostic evaluations of chronic hepatitis. Liver biopsy is considered the definitive investigation for assessing the stage of liver fibrosis but it carries several limitations. FIB-4 and APRI also have a limited accuracy. The National Committee for Control of Viral Hepatitis $(\mathrm{NCCVH})$ in Egypt has supplied a valuable pool of electronic patients' data that data mining techniques can analyze to disclose hidden patterns, trends leading to the evolution of predictive algorithms.
\end{abstract}

\begin{abstract}
Aim: to collaborate with physicians to develop a novel reliable, easy to comprehend noninvasive model to predict the stage of liver fibrosis utilizing routine workup, without imposing extra costs for additional examinations especially in areas with limited resources like Egypt.

Methods: This multi-centered retrospective study included baseline demographic, laboratory, and histopathological data of 69106 patients with chronic hepatitis $C$. We started by data collection preprocessing, cleansing and formatting for knowledge discovery of useful information from Electronic Health Records EHRs. Data mining has been used to build a decision tree (Reduced Error Pruning tree (REP tree)) with 10-fold internal cross-validation. Histopathology results were used to assess accuracy for fibrosis stages. Machine learning feature selection and reduction (CfsSubseteval / best first) reduced the initial number of input features $(N=15)$ to the most relevant ones $(N=6)$ for developing the prediction model.
\end{abstract}

Results: In this study, 32419 patients had F(0-1), 25073 had F(2) and 11615 had F(3-4). FIB-4 and APRI revalidation in our study showed low accuracy and high discordance with biopsy results, with overall AUC 0.68 and 0.58 respectively. Out of 15 attributes machine learning selected Age, AFP, AST, glucose, albumin, and platelet as the most relevant attributes. Results for REP tree indicated an overall classification accuracy up to $70 \%$ and ROC Area 0.74 which was not nearly affected by attribute reduction, and pruning . However attribute reduction, and 
tree pruning were associated with simpler model easy to understand by physician with less time for execution.

Conclusion: This study we had the chance to study a large cohort of 69106 chronic hepatitis patients with available liver biopsy results to revise and validate the accuracy of FIB-4 and APRI. This study represents the collaboration between computer scientist and hepatologists to provide clinicians with an accurate novel and reliable, noninvasive model to predict the stage of liver fibrosis.

\section{KEYWORDS}

Liver Fibrosis, Data Mining, Weka, Decision Tree, Attribute Reduction, Tree Pruning.

\section{INTRODUCTION}

Hepatitis $\mathrm{C}$ virus (HCV) is a worldwide etiology of chronic hepatic infection particularly in Egypt where genotype 4 being responsible for $>90 \%$ of cases, and the remaining is due to genotype-1 [1,2]. The natural history of chronic hepatitis $\mathrm{C}$ (CHC) infection passes through consecutive steps of progressive fibrosis, hepatic cirrhosis that decompensates into end-stage liver disease and the dismal malignancy of Hepatocellular carcinoma (HCC) [3]. The gold standard for staging liver fibrosis remains percutaneous liver biopsy, which is an invasive procedure [4]. Fibroscan ultrasonography is non-invasive and reliable methods for diagnosis of the stage of liver fibrosis, compared to liver biopsy [5]. The Aspartate aminotransferase-toplatelet ratio index (APRI), and FIB-4 scores are simple, noninvasive, easy to perform, inexpensive and reproducible algorithms for diagnosing advanced fibrosis stages [5-7], but they are not consistently accurate in classifying fibrosis stages [8].

\subsection{Motivation AND AIM}

Medicine involves decision making and classification or prediction is an important part of it. However, medical classification or prediction is usually a very complex and hard process. Human reasoning even of expert clinician could not deal with highly dimensional criteria of health care data, their underlying associations, and predictive capability. In Egypt, the NCCVH program has provided a valuable pool of demographic and laboratory data which can lead to information discovery, making good use of the data stored in EHRs of CHC patients and the conducting large population based researches.

Most of medical studies rely on statistical analysis and to a much less extent on machine learning. Even those studies which consider machine learning are usually defective in the medical aspects and are complex or difficult to comprehend by physicians. This study represents a multidisciplinary approach between hepatologists and health informatics researchers to develop a novel, reliable, and non-invasive model to predict the stage of liver fibrosis based on routine workup, without imposing extra costs for additional examinations especially in areas with limited resources like Egypt. Further we use machine learning attribute reduction and decision tree pruning to simplify the model to be easily understood by physicians.

\subsection{Our Contribution}

I. To our knowledge this study enrolled the largest sample size of $\mathrm{CHC}$ patients with available histopathological results. This chance will not be repeated with the new era of directly acting antiviral therapy which did not necessitate liver biopsy. 
II. This study represents collaboration between physicians and medical informatics people to overcome the complexity of Machine Learning algorithm, and to provide easy to comprehend model using ML tools as feature reduction, and decision tree pruning.

III. This study provides a chance to re-validate the diagnostic accuracy of the famous and widely used FIB-4 and APRI. Further we use the power of data mining to explore this large volumes of data stored in electronic health records to discover hidden patterns and relationships to provide more accurate model for prediction of advanced liver fibrosis.

\subsection{RELATED Work}

Data mining is a reliable method of predictive analysis which explores tremendous volumes of data to discover hidden patterns and relationships in highly complex dataset. Data mining differs from classical statistical analysis in that statistical inference in its hypothesis testing sense may not be appropriate, and the questions asked may not be applicable to large datasets. In most applications of data mining, there is no a priori reason to sample and all attributes of data is readily available for exploratory questions. Thus, it can enable the development of predictive models.

Decision trees are the recommended approach for building comprehensible clear predictive models, which are simple and quick to build with adequate accuracy. Conversion of decision trees into classification rules is easy. They do not require any domain knowledge and easy to assimilate by physicians. The main benefit of decision trees over logistic regression analysis is that decision trees are easy to understand [9]. The simple allocation of patients into subgroups by following the flowchart design could define the anticipated possibility of outcome [10].Prognostic factors of different diseases have been defined using decision trees.

In previous work we implemented Data mining analysis explores data, and trends that enable the development of models to diagnose HCC [11, 12], the prediction of therapeutic outcome of HCV patients utilizing simple laboratory data [13]. We further addressed the issue of performance evaluation of decision tree classifiers, where we assessed the correctly classified instances, recall, precession, and area under the curve [14]. Hashem, et al. used the decision tree learning algorithm to provide an accurate prediction of advanced Liver fibrosis in CHC [15].

\section{Patients And Methods}

This retrospective multi-center study included 69106 Egyptian patients with CHC who were naive candidates for antiviral therapy registered by The Egyptian National Committee for Control of Viral Hepatitis in the period from January 2010 till December 2014. A local ethical committee approval was available before starting data collection. With respect to patient's confidentiality, all patients were represented in the study by code numbers after concealing all their personal data. The protocol of the study conformed to the ethical guidelines of the 1975 Declaration of Helsinki. Knowledge discovery of useful information from Electronic Health Records EHRs of a cohort of 
69106 patients necessitates putting high quality data. characterized by accuracy, integrity, completeness, validity, consistency, uniformity and uniqueness.

This is a multistep process, composed of the following primary activities:

- Data collection pre-processing, cleansing and formatting.

- Data auditing and statistical analysis.

- Feature selection and reduction to minimize the dimensionality of the problem.

- Selecting appropriate mathematical models to represent the trends.

- Evaluating the selected models.

\subsection{Data Collection, Cleansing and Formatting}

Knowledge discovery of useful data from EHRs of a standardized recruitment questionnaire was completed by $\mathrm{CHC}$ patients physicians that included demographic features (age, gender, body mass index (BMI), Complete blood count (CBC), Liver biochemical testing (alanine aminotransferase (ALT), aspartate aminotransferase (AST), alkaline phosphatase, Serum bilirubin, albumin and INR), blood glucose, and Serum creatinine and alpha fetoprotein (AFP), thyroid stimulating hormone (TSH), antinuclear antibody (ANA), and HCV quantitative real time polymerase chain reaction (Abbot Real Time, detection limit 30 (IU/ml). In addition to their histopathological data where liver biopsy was mandatory for fibrosis assessment in the interferon era. Metavir scoring system of fibrosis F0-F4 was used as a gold standard to assess accuracy for the stage of fibrosis.

The information was largely collected from medical notes and checked for completeness and correctness. Finally, data were entered onto a computer database (in the form of excel sheets) by a person with paramedical training then it was transformed to a standard relational database management system. All laboratories undergone proper quality control measures to ensure validity of their results. Data cleansing is detecting and adjusting (or removing) corrupt or inaccurate patients' records from a record set in addition to excluding of typographical errors or validating and correcting values against a known list of entities. If inconsistencies were found or necessary corrections were needed, the form/s was returned to the physician for revision. The identified inappropriate parts of the data may be replaced, modified or deleted. High quality data needs to be characterized by accuracy, integrity, completeness, validity, consistency, uniformity and uniqueness. Data transformation techniques were used to format and prepare the patient records to be processed by the learning algorithms. Each attribute was classified as numerical or categorical then validated, to setup high quality valid consistent data.

\subsection{Data Auditing And Statistical Analysis}

Numerical data are reported as means \pm standard deviation (S.D). Categorical data are represented as counts and percentages. The student t-test and the Chi-square test are used when appropriate. Statistical significance is considered if the probability of occurrence by chance is $5 \%$ or less $(\mathrm{P}<0.05)$. Statistical analysis was used for cross tabulation, and estimation of sensitivity, specificity, positive predictive value, negative predictive value and ROC curves. 


\subsection{Feature Selection AND REDUCTION}

In this stage we aim to reduce the initial number of input features $(\mathrm{N}=15)$ to the most relevant ones $(\mathrm{N}=6)$ for developing the prediction model. Attributes that showed statistically significant correlation with F2-F4 stages of fibrosis were used to create a decision tree to predict liver fibrosis stage. However, using such statistical based approaches for feature selection may impair classification performance. To avoid the limitations of statistical based techniques for feature selection, we used correlation based Feature Sub-set Selection for Machine Learning (CfsSubsetEval) to evaluate the worth of a subset of attributes through considering the individual predictive ability of each feature along with the degree of redundancy between them. The second step is searching the space of attribute subsets by greedy hill climbing augmented with a backtracking facility. Best first may start with the empty set of attributes (forward search), or start with the full set of attributes (backward search), or start search at any point in both directions [16].

\subsection{Selecting Appropriate Mathematical Models to Represent the TrendS}

Reduced Error pruning tree (REP tree) is considered a fast decision tree learner that builds a decision/regression flowchart using information gain/variance and prunes it using reduced error pruning. The Weka program can be used for testing data sets using a variety of open source Machine Learning algorithms. The workbench includes methods for all the standard data mining problems e.g. clustering, association rule mining, regression, classification, and attribute selection. In addition, it involves a variety of tools for converting, pre-processing datasets, feed it into a learning scheme, and analyse the resulting classier and its performance. Weka is available from http://www.cs.waikato.ac.nz/ml/weka.

\subsection{Performance Evaluating the Selected Models}

The performance of decision trees built using all attributes was compared to those built after attribute reduction. The calculated algorithm was validated using the k-fold cross-validation approach. In brief, we divided the original sample into $\mathrm{k}$ sub-samples. The cross-validation process was repeated $\mathrm{k}$ times (folds). Each of the $\mathrm{k}$ sub-samples was used once as the validation data [17]. We assessed the performance of algorithm according to evaluation matrix based on values for the correctly classified instance, precision (specificity), recall (sensitivity), and Receiver operating characteristic (ROC) curve.

\section{RESUlts}

To our knowledge this multi-centered registry study has the largest sample size (69106 HCV patients) with liver biopsy histo-pathological results. The patients were divided according to their Metavir scores into, 32419 patients $(46.9 \%)$ with minimal fibrosis stages (F0-F1) and F2 in 25073 patients (36.3\%), and advanced fibrosis (F3-F4) in 11615 patients (16.8\%). The demographic and laboratory features and statistical differences between the studied groups (F0F2 / F3-F4) are shown in Table 1 arranged according to their significance. Male patients 
represented $71.8 \%$ (49618 patients) of the study population. There was a statistically significant difference between the 2 groups in terms of their age, body mass index (BMI), platelet count, white blood cell count(WBCs), AFP, AST, serum bilirubin, albumin and alkaline phosphatase. All the 15 attributes used for liver fibrosis prediction were presented as categorical or numerical, and arranged according to statistical significance (p-value) and their weight as shown in Table 2. Using machine learning attribute selection these attributes were reduced to six attributes namely Age, AFP, AST, glucose, albumin, and platelet that proved to be the most relevant attributes.

In our study, FIB-4 and APRI diagnosed advanced fibrosis (F3-F4) with a good negative predictive value and high specificity which is comparable to the original study, but low positive predictive value and with poor sensitivity. Patients with advanced fibrosis were not diagnosed and there was a great discordance between FIB-4 and APRI compared to liver biopsy results. The correctly classified F3-F4 patients were $57.5 \%$ and $13.9 \%$ for cut-off value $\geq 1.45$ and $\geq 3.25$ receptively. The results of APRI test showed poor diagnostic abilities and only $32.7 \%$ F3-F4 patients were correctly classified at cut off value $\geq 1$.

This high discordance in our study between the actual results of liver biopsy and the other two tools APRI and FIB-4 with overall AUC 0.58 (Figure 1) and 0.68 (Figure 2) respectively, has provided us the motivation of using data mining techniques to find a new non-invasive predictive algorithm. Machine learning techniques such as Decision Trees (classification trees) have been used for prediction, classification, and as diagnostic tools.

able 1. Baseline characteristics of the study population.

\begin{tabular}{|c|c|c|c|}
\hline Attribute & F 0-1 & F 2-4 & P-value \\
\hline Age & $37.31 \pm .073$ & $43.13 \pm .067$ & .0001 \\
\hline BMI & $26.11 \pm .02$ & $27.04 \pm .02$ & .0001 \\
\hline Platelets & $221.14 \pm .34$ & $203.95 \pm .34$ & .0001 \\
\hline AFP & $5.99 \pm .14$ & $9.60 \pm .20$ & .0001 \\
\hline $\begin{array}{c}\text { Fasting blood glucose } \\
\text { (mg/dL) }\end{array}$ & $102.0 \pm 27.9$ & $110.6 \pm 38.0$ & .001 \\
\hline Total Bilirubin (mg/dl) & $0.84 \pm .02$ & $1.47 \pm .34$ & .002 \\
\hline Albumin (g/dL) & $4.38 \pm .002$ & $4.30 \pm .002$ & .003 \\
\hline AST (IU/L) & $50.44 \pm .35$ & $51.52 \pm 1.47$ & .018 \\
\hline WBC (x1000) & $6.4 \pm .01$ & $6.44 \pm .01$ & .028 \\
\hline $\begin{array}{c}\text { Alkaline Phosphatase } \\
\text { (IU/L) }\end{array}$ & $113.54 \pm .41$ & $118.31 \pm 1.68$ & .035 \\
\hline $\begin{array}{c}\text { Serum Creatinine } \\
\text { (mg/dl) }\end{array}$ & $0.85 \pm 0.2$ & $1.04 \pm 0.4$ & .200 \\
\hline Hb (G/L) & $13.87 \pm .009$ & $13.89 \pm .008$ & .340 \\
\hline ALT (IU/L) & $86.51 \pm 29.4$ & $118.87 \pm 63.31$ & .371 \\
\hline HCV RNA (IU/M) & $22 \times 10^{5} \pm 27 \times 10^{4}$ & $24 \times 10^{5} \pm 40 \times 10^{4}$ & .442 \\
\hline
\end{tabular}


Table 2. Features selection for prediction of advanced fibrosis F3-4.

\begin{tabular}{|c|c|c|c|c|c|}
\hline Attribute & $\begin{array}{c}\text { Represented } \\
\text { as }\end{array}$ & $\begin{array}{c}\text { Statistical } \\
\text { sig } \\
(\text { P-Value })\end{array}$ & $\begin{array}{c}\text { Attribute } \\
\text { weight }\end{array}$ & $\begin{array}{c}\text { ML } \\
\text { selection }\end{array}$ \\
\hline 1 & Gender & Categorical & .021 & 0.00243 & No \\
\hline 2 & Age & Numeric & .0001 & 0.05968 & Yes \\
\hline 3 & BMI & Numeric & .0001 & 0.02108 & No \\
\hline 4 & Hb (G/L) & Numeric & .340 & 0.00153 & No \\
\hline 5 & WBC (x1000) & Numeric & .028 & 0.00129 & No \\
\hline 6 & Platelets & Numeric & .0001 & 0.05351 & Yes \\
\hline 7 & $\begin{array}{c}\text { Total Bilirubin } \\
\text { (mg/dl) }\end{array}$ & Numeric & .002 & 0.02191 & No \\
\hline 8 & Albumin (g/dL) & Numeric & .003 & 0.01682 & Yes \\
\hline 9 & AST (IU/L) & Numeric & .018 & 0.02399 & Yes \\
\hline 10 & ALT (IU/L) & Numeric & .371 & 0.02456 & No \\
\hline 11 & $\begin{array}{c}\text { Alkaline Phosphatase } \\
\text { (IU/L) }\end{array}$ & Numeric & .035 & 0.00475 & No \\
\hline 12 & $\begin{array}{c}\text { Fasting blood } \\
\text { glucose (mg/dL) }\end{array}$ & Numeric & .0001 & 0.01436 & Yes \\
\hline 13 & AFP & Numeric & .0001 & 0.06157 & Yes \\
\hline 14 & HCV RNA (IU/M) & Numeric & .442 & 0.00773 & No \\
\hline 15 & $\begin{array}{c}\text { Serum Creatinine } \\
\text { (mg/dl) }\end{array}$ & Numeric & .043 & 0.00191 & No \\
\hline
\end{tabular}

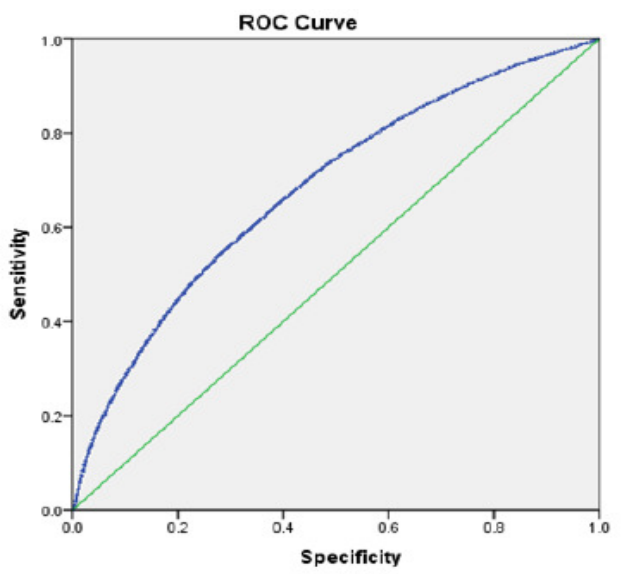

Figure 1. FIB-4 ROC Curve

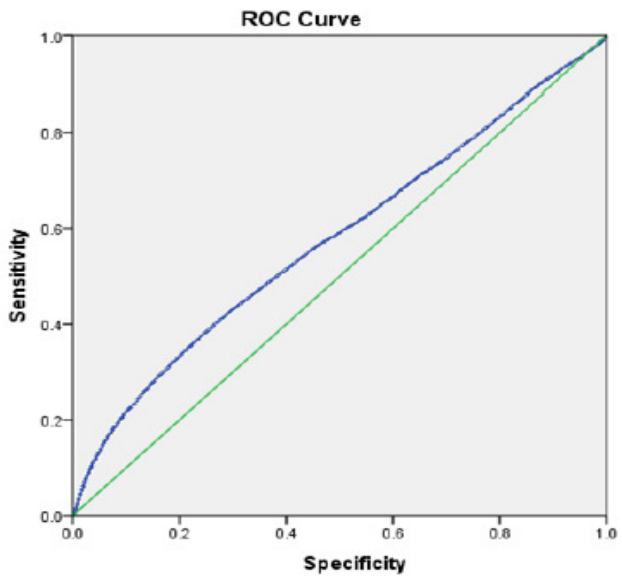

Figure 2. APRI ROC Curve

At first we applied REPTree as a predictive algorithm using all the 15 attributes. This decision tree model showed that AFP level was selected as the variable of initial split (most decisive), with optimal cut-off value of $\leq 6.55 \mathrm{ng} / \mathrm{mL}$. Age is the next decisive splitting attribute with optimal cut-off value of $\leq 38.5$ years, platelet count with optimal cut-off value of $\leq 181.5$, other attributes as AST, ALT, glucose, BMI, albumin, bilirubin, and INR have less decisive role for prediction of 
fibrosis (Figure 4). Patients with significant liver fibrosis were significantly older in age, higher AFP levels and lower platelet counts.

Results indicated an overall classification accuracy about $67 \%$ and ROC Area 0.74 for REPtree (Figure 3), which was not affected by attribute reduction. This model was further simplified by attribute reduction using only the six attributes selected using machine learning as shown in table 2. The diagnostic performance analysis of advanced fibrosis F3F4 of the various prediction models clearly shows that the diagnostic power of REPtree over exceeds the two popular models APRI and FIB-4 (Table 3). This simple easy to comprehend prediction model could be provided to doctors so they can know the probability of stage of fibrosis by entering simple data as age, AFP, and platelet.

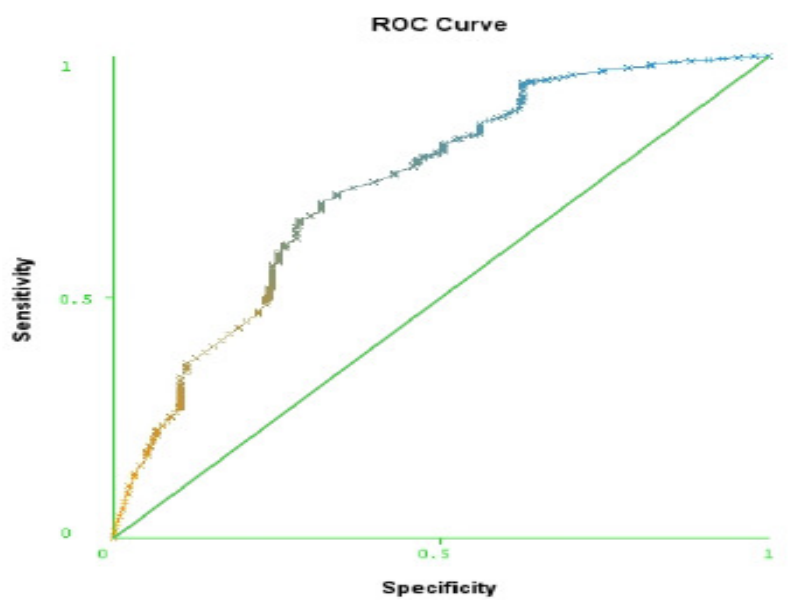

Figure 3. FIB-4 ROC Curve

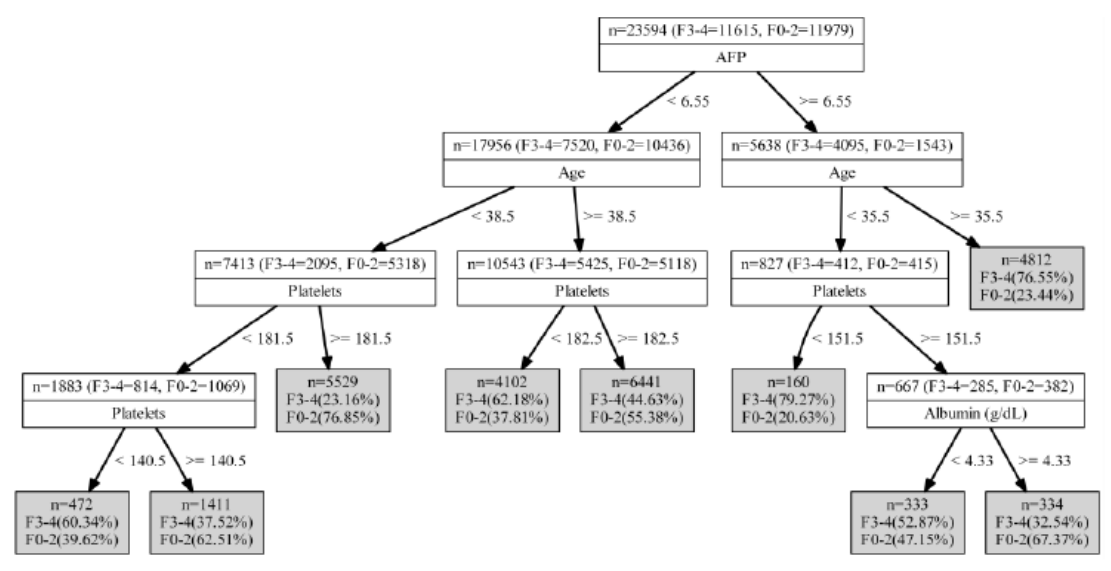

Figure 4. Decision tree (REPTree) to predict liver fibrosis. 
Table 3. Diagnostic performance of advanced fibrosis F3-4 prediction models.

\begin{tabular}{|c|c|c|c|c|c|c|c|}
\hline & $\begin{array}{c}\text { REPTr } \\
\text { ee }\end{array}$ & $\begin{array}{c}\text { REPTree } \\
\text { pruned }\end{array}$ & REPTree & $\begin{array}{c}\text { REPTree } \\
\text { pruned }\end{array}$ & $\begin{array}{c}\text { FIB-4 } \\
\mathbf{> 1 . 4 5}\end{array}$ & $\begin{array}{c}\text { FIB-4 } \\
\mathbf{> 3 . 2 5}\end{array}$ & $\begin{array}{c}\text { APRI } \\
>\mathbf{1}\end{array}$ \\
\hline Attributes & 15 & 15 & 6 & 6 & $\mathbf{4}$ & 4 & 2 \\
\hline Time(sec) & 1.68 & 0.21 & 0.6 & 0.1 & - & - & - \\
\hline Tree Size & 88.3 & 17 & 875 & 17 & - & - & - \\
\hline $\begin{array}{c}\text { Correctly } \\
\text { Classified\% }\end{array}$ & $68.1 \%$ & $70 \%$ & $66.4 \%$ & $67.3 \%$ & $57.5 \%$ & $13.9 \%$ & $32.7 \%$ \\
\hline TP Rate & $66.4 \%$ & $61.9 \%$ & $62.6 \%$ & $60.8 \%$ & $27.62 \%$ & $45.16 \%$ & $25.13 \%$ \\
\hline FP Rate & $30 \%$ & $28.3 \%$ & $29.9 \%$ & $26.5 \%$ & $88.55 \%$ & $84.30 \%$ & $85.81 \%$ \\
\hline Precision & $68.1 \%$ & $68 \%$ & $67 \%$ & $69 \%$ & $68.58 \%$ & $96.48 \%$ & $80.68 \%$ \\
\hline Recall & $66.4 \%$ & $61.9 \%$ & $62.6 \%$ & $60.8 \%$ & $57.48 \%$ & $13.89 \%$ & $32.70 \%$ \\
\hline ROC Area & 0.742 & 0.732 & 0.732 & 0.731 & 0.68 & 0.68 & 0.58 \\
\hline
\end{tabular}

\section{Discussion AND CONCLUSION}

Staging of Liver fibrosis is an integral component in the appropriate management of $\mathrm{CHC}$ and the prediction of patient prognosis. Patients with advanced fibrosis (F3-F4) will eventually progress to cirrhosis carrying the risk of liver decompensation, primary liver cancer of hepatocellular carcinoma and death [18]. The needed evaluation and follow up of liver fibrosis stage have been traditionally performed by liver biopsy [19]. There have been many proposed non-invasive biomarkers, an ideal serological test for assessing liver fibrosis stages is still awaited [18].

Liver biopsy is still the gold standard for assessing the staging of liver fibrosis. The collected demographic, biochemical, and histological data sets for a large cohort of 69106 chronic hepatitis patients with available liver biopsy results give us the chance to revise and validate the accuracy of FIB-4 and APRI [4, 20]. In our study, FIB-4 and APRI diagnosed advanced fibrosis (F3F4with a good negative predictive value and high specificity which is comparable to the original study, but low positive predictive value and with poor sensitivity. Patients with advanced fibrosis were not diagnosed and there was a great discordance between FIB-4 and APRI compared to liver biopsy results.

This high discordance in our study between biopsy, FIB-4 and APRI with overall AUC 0.69 and 0.58 provided the motivation to use data mining techniques to find a noninvasive predictive algorithm. Machine learning techniques such as Decision Trees (classification trees) have been used for prediction, classification, and as diagnostic tools. The decision tree method is a potent statistical technique for allocation, prediction, and, interpretation that has a lot of applications in the field of medical research. The relationship between data is represented in an easy to follow top down tree architecture. The root node of the decision tree is the most influential piece of data that affects the response variable in the model [21, 22]. It is simple, easy to interpret and can efficiently deal with large, complicated data without a complicated parametric structure [23]. An alternative approach to create a decision tree is to grow a large tree, and then prune it by 
removing nodes that provide less additional information. Pruning is usually performed for enhancing tree comprehensibility while maintaining (or even improving) its accuracy [24]. Reduced error pruning (REP) is a conceptually simple strategy that should be performed in a bottom-up fashion $[14,17]$.

Conclusion: This is the largest multi-centered registry study including the largest sample size of CHC patients (69106 patients). This large sample size could explain the discrepancy between these study findings and the reports from the original studies or other studies that included smaller sample size (hundreds). This large sample size showed a great discordance between FIB4, APRI and Metavir score. Decision tree (REP tree) could provide a reliable method for noninvasive diagnosis of fibrosis stages using routine laboratory parameters

\section{ACKNOWLEDGMENT}

The authors would like to thank Egyptian National Committee for Control of Viral Hepatitis, Ministry of Health and Population (MOHP) for supplying the patient's data.

\section{REFERENCES}

[1] I. Waked, W. Doss, M. H. El-Sayed, C. Estes, H. Razavi, G. Shiha, A. Yosry, and G. Esmat, "The current and future disease burden of chronic hepatitis c virus infection in egypt," Arab J Gastroenterol, vol. 15, no. 2, pp. 45-52, 2014.

[2] S. Blach, S. Zeuzem, M. Manns, I. Altraif, A.-S. Duberg, D. H. Muljono, I. Waked, S. M. Alavian, M.-H. Lee, and F. Negro, "Global prevalence and genotype distribution of hepatitis c virus infection in 2015: a modelling study," The Lancet Gastroenterology \& Hepatology, vol. 2, no. 3, pp. 161-176, 2017.

[3] E. A. for the Study of the Liver, "Easl recommendations on treatment of hepatitis c 2014," Journal of hepatology, vol. 61, no. 2, pp. 373- 395, Aug 2014, IR: 20160113; JID: 8503886; 0 (Antiviral Agents); 0 (RNA, Viral); 2014/05/14 06:00 [entrez]; 2014/05/14 06:00 [pubmed]; 2015/08/12 06:00 [medline]; ppublish.

[4] V. Papastergiou, E. Tsochatzis, and A. K. Burroughs, "Non-invasive assessment of liver fibrosis," Annals of gastroenterology, vol. 25, no. 3, pp. 218-231, 2012, 1R: 20170224; JID: 101121847; OTO: NOTNLM; 2011/02/27 00:00 [received]; 2012/03/13 00:00 [accepted]; 2014/04/10 06:00 [entrez]; 2012/01/01 00:00 [pubmed]; 2012/01/01 00:00 [medline]; ppublish.

[5] M. Alboraie, M. Khairy, M. Elsharkawy, N. Asem, A. Elsharkawy, and G. Esmat, "Value of egyscore in diagnosis of significant, advanced hepatic fibrosis and cirrhosis compared to aspartate aminotransferaseto- platelet ratio index, fib4 and forns' index in chronic hepatitis c virus," Hepatology Research, vol. 45, no. 5, pp. 560-570, 2015.

[6] M. Khairy, M. Abdel-Rahman, M. El-Raziky, W. El-Akel, N. Zayed, H. Khatab, and G. Esmat, "Non-invasive prediction of hepatic fibro- sis in patients with chronic hcv based on the routine pretreatment workup," Hepatitis monthly, vol. 12, no. 11, p. e6718, Nov 2012, IR: 20130530; JID: 101277874; OTO: NOTNLM; 2012/06/09 00:00 [received]; 2012/07/19 00:00 [revised]; 2012/08/13 00:00 [accepted]; 2013/01/25 06:00 [entrez]; 2013/01/25 06:00 [pubmed]; 2013/01/25 06:01 [medline]; ppublish.

[7] A. Yosry, R. Fouad, S. A. Alem, A. Elsharkawy, M. El-Sayed, N. Asem, E. Hassan, A. Ismail, and G. Esmat, "Fibroscan, apri, fib4, and guci: Role in prediction of fibrosis and response to therapy in egyptian patients with hcv infection," Arab Journal of Gastroenterology, vol. 17, no. 2, pp. 78-83, 2016.

[8] T. G. Ragazzo, D. Paranagua-Vezozzo, F. R. Lima, D. F. de Campos Mazo, M. G. Pessoa, C. P. Oliveira, V. A. F. Alves, and F. J. Carrilho, “Accuracy of transient elastography-fibroscan, acoustic radiation force impulse (arfi) imaging, the enhanced liver fibrosis (elf) test, apri, and the fib-4 index 
compared with liver biopsy in patients with chronic hepatitis c," Clinics, vol. 72, no. 9, pp. 516-525, 2017.

[9] I. H. Witten, E. Frank, M. A. Hall, and C. J. Pal, Data Mining: Practical machine learning tools and techniques. Morgan Kaufmann, 2016.

[10] M. LeBlanc and J. Crowley, A review of tree-based prognostic models, ser. Recent advances in clinical trial design and analysis. Springer, 1995, pp. 113-124.

[11] D. A. E. H. Omran, A. H. Awad, M. A. El, R. Mabrouk, A. F. Soliman, and A. O. A. Aziz, "Application of data mining techniques to explore predictors of hec in egyptian patients with hcvrelated chronic liver," Asian Pacific Journal of Cancer Prevention, vol. 16, no. 1, pp. 381-385, 2015.

[12] A. Abdelaziz, A. Awad, H. Shousha, M. Mahmoud, D. Om- ran, A. h. K. Abdelmaksoud, and M. Mabrouk, "Meta-learning analysis to find the best predictive algorithm for prediction of hepatocellular carcinoma outcome in a cohort of 1200 hcv-related patients," in The European Association for the Study of the Liver (EASL) - HCC summit, vol. P13.02-YI, 02 2017. [Online]. Available: http://livertree.easl.eu/easl/2017/geneva/165849/hend.shousha. metalearning.analysis.to.find.the.best.predictive.algorithm.for.html

[13] M. E. Raziky, W. F. Fathalah, Z. Zakaria, H. G. Eldeen, A. Abul- Fotouh, A. Salama, A. Awad, G. Esmat, and M. Mabrouk, "Predictors of virological response in 3,235 chronic hcv egyptian patients treated with peginterferon alpha-2a compared with peginterferon alpha-2b using statistical methods and data mining techniques," Journal of Inter- feron \& Cytokine Research, vol. 36, no. 5, pp. 338$346,2016$.

[14] A. Awad, M. Mabrouk, T. Awad, N. Zayed, S. Mousa, and M. Saeed, "Performance evaluation of decision tree classifiers for the prediction of response to treatment of hepatitis c patients," in Proceedings of the 8th International Conference on Pervasive Computing Technologies for Healthcare. ICST (Institute for Computer Sciences, Social-Informatics and Telecommunications Engineering), 2014, pp. 186-190.

[15] S. Hashem, G. Esmat, W. Elakel, S. Habashy, S. A. Raouf, S. Darweesh, M. Soliman, M. Elhefnawi, M. El-Adawy, and M. ElHefnawi, "Accurate prediction of advanced liver fibrosis using the decision tree learning algorithm in chronic hepatitis c egyptian patients," Gastroenterology research and practice, vol. 2016, 2016.

[16] M. Hall, "Correlation-based feature subset selection for machine learn- ing," Thesis submitted in partial fulfillment of the requirements of the degree of Doctor of Philosophy at the University of Waikato, 1998.

[17] M. Bal, M. F. Amasyali, H. Sever, G. Kose, and A. Demirhan, "Per- formance evaluation of the machine learning algorithms used in inference mechanism of a medical decision support system," The Scientific World Journal, vol. 2014, p. 137896, 2014, IR: 20151029; JID: 101131163; 2014/06/04 00:00 [received]; 2014/08/07 00:00 [revised]; 2014/08/20 00:00 [accepted]; 2014/10/09 06:00 [entrez]; 2014/10/09 06:00 [pubmed]; 2015/06/24 06:00 [medline]; ppublish.

[18] N. Yada, M. Kudo, N. Kawada, S. Sato, Y. Osaki, A. Ishikawa, H. Miyoshi, M. Sakamoto, M. Kage, and O. Nakashima, "Noninvasive diagnosis of liver fibrosis: utility of data mining of both ultrasound elastography and serological findings to construct a decision tree," Oncology, vol. 87, no. Suppl. 1, pp. 63-72, 2014.

[19] Y. Lurie, M. Webb, R. Cytter-Kuint, S. Shteingart, and G. Z. Lederkremer, "Non-invasive diagnosis of liver fibrosis and cirrhosis," World journal of gastroenterology, vol. 21, no. 41, pp. 11 567-11 583, Nov 7 2015, IR: 20170220; JID: 100883448; 0 (Biomarkers); OTO: NOTNLM; 2015/04/28 00:00 [received]; 2015/07/23 00:00 [revised]; 2015/09/15 00:00 [accepted]; 2015/11/12 06:00 [entrez]; 2015/11/12 06:00 [pubmed]; 2016/11/01 06:00 [medline]; ppublish.

[20] M. Adler, B. Gulbis, C. Moreno, S. Evrard, G. Verset, P. Golstein, B. Frotscher, N. Nagy, and P. Thiry, "The predictive value of fib4 versus fibrotest, apri, fibroindex and forns index to noninvasively 
estimate fibrosis in hepatitis c and nonhepatitis c liver diseases," Hepatology, vol. 47, no. 2, pp. 762763, 2008.

[21] T. Poynard, Y. Ngo, H. Perazzo, M. Munteanu, P. Lebray, J. Mous- salli, D. Thabut, Y. Benhamou, and V. Ratziu, "Prognostic value of liver fibrosis biomarkers: a meta-analysis," Gastroenterology \& hepatology, vol. 7, no. 7, pp. 445-454, Jul 2011, IR: 20170220; JID: 101262648; OTO: NOTNLM; 2012/02/03 06:00 [entrez]; 2012/02/03 06:00 [pubmed]; 2012/02/03 06:01 [medline]; ppublish.

[22] E. Rezaei-Darzi, F. Farzadfar, A. Hashemi-Meshkini, I. Navidi, M. Mahmoudi, M. Varmaghani, P. Mehdipour, M. S. Alamdari, B. Tayefi, and S. Naderimagham, "Comparison of two data mining techniques in labeling diagnosis to iranian pharmacy claim dataset: artificial neural network (ann) versus decision tree model.” Archives of Iranian Medicine (AIM), vol. 17, no. 12, 2014.

[23] Y. Y. Song and Y. Lu, "Decision tree methods: applications for classification and prediction," Shanghai archives of psychiatry, vol. 27, no. 2, pp. 130-135, Apr 25 2015, IR: 20170220; JID: 9891453; OTO: NOTNLM; 2015/04/01 00:00 [received]; 2015/04/09 00:00 [accepted]; 2015/06/30 06:00 [entrez]; 2015/06/30 06:00 [pubmed]; 2015/06/30 06:01 [medline]; ppublish.

[24] R. C. Barros, A. T. Winck, K. S. Machado, M. P. Basgalupp, A. C. de Carvalho, D. D. Ruiz, and O. N. de Souza, "Automatic design of decision-tree induction algorithms tailored to flexible-receptor docking data," BMC bioinformatics, vol. 13, no. 1, p. 310, 2012. 